\title{
End anchor effects in the discriminability of physical and social stimuli'
}

HENRY MILLER, UNIVERSITY OF CALIFORNIA, BERKELEY JAMES BIERI, UNIVERSITY OF TEXAS

\begin{abstract}
Abstraet
Discriminability of stimuli was reduced when the range being judged contained less salient end anchors. These effects were observed in both physical and social stimulus domains. Lowest discriminability occurred when no salient end stimuli were present. Social stimuli in all conditions were discriminated less well than physical stimuli.
\end{abstract}

Problem

One of the factors assumed to affect the discriminability of stimuli is the relative saliency of the end stimuli in the given range or series being judged. Johnson \& King (1964), for example, have demonstrated that when natural end stimuli are present as in a bipolar continuum ranging from all blue dots to all green dots, discrimination is better than when end stimuli are less salient (e. g., some mixture of blue and green dots). Such salient end stimuli operate as anchors, presumably improving discrimination of adjacent stimuli. In the area of clinical judgement, Miller \& Bieri (1963) and Hunt, Schwartz, \& Walker (1965) have reported that extreme social stimuli a re judged less variably than are mid-range stimuli.

The present study extends this analysis by comparing the effects upon the discriminability of social and physical stimuli of ranges which vary in terms of the relative saliency of end anchors. In such a study, it is necessary to equate the relative saliency of end anchors in both stimulus realms on some rational, a priori grounds. For example, it is evident that among sensory continua, the relative saliency of end stimuli may vary. In contrast to the unequivocally salient end stimuli in the Johnson and King study, a continuum such as length has less salient end stimuli. If a dimension ranges from stimuli of $1 / 4$ in to 4 in, for example, it is likely that the shorter end stimulus will provide a more salient end anchor than the longer end stimulus, due in part to its recognition as a $1 / 4$ in unit and the overlearned tendency to perceive longer lengths as multiples of the shorter lengths. Thus, the longer lengths will presumably offer less clearcut possibilities as salient end anchors.

If we consider social or behavioral dimensions, we might also expect differences in the relative saliency of the end stimuli in any given range. It would seem that in most behavioral dimensions the high intensity end of the continuum offers a better or more salient end anchor than does the low end of the continuum. For example, a highly aggressive behavior would be a more salient end stimulus than a low aggressive behavior. A very low ag- gressive behavior may in fact be perceived as a relatively neutral or indifferent point on a bipolar dimension ranging from extreme aggression to extreme friendliness or love.

These considerations lead us to expect that a physical continuum such as length and a behavior continuum such as aggression will have comparable formal characteristics in terms of the relative saliency of end stimuli as anchors. We would expect, then, that in either stimulus realm, a range containing the more salient end stimulus would be better discriminated than a range containing a less salient end stimulus. Further, we would expect that a range containing no salient end stimuli, as exemplified by mid-range stimuli, should be least well discriminated.

\section{Method}

Four conditions of range were generated within each stimulus realm. Sixteen previously scaled items of aggressive behavior ranging from low to extreme aggression and separated by approximately equal intervals were available from previous research (Bieri, Orcutt \& Leaman, 1963). Sixteen lengths of lines were constructed ranging from a short end stimulus of $1 / 4$ in to a long end stimulus of 4 in. Each stimulus was presented on a card to a judge who was asked to rate the stimulus on a scale from 1 (low aggression or shortest length) to 8 (high aggression or longest length). Eight stimuli were selected from the 16 to form four conditions of range. Range I consisted of the 8 stimuli from the low aggression (or short) end of the continuum. Range II consisted of the 8 stimuli from the high aggression (or long) end of the dimension. Both these ranges contain one salient end stimulus; however, for the social continuum the greatest saliency of end stimulus is in Range II, and for the physical continuum it is in Range I. Range III consisted of the 8 stimuli from the middle of each continuum and was considered to offer no salient end stimuli. Finally, Range IV included 8 stimuli sampling the entire continuum, and would thus contain two salient end stimuli.

Fifteen judges were randomly assigned to each of the four range conditions, yielding a totäl of 60 judges. For the assigned range, each judge rated both the social and physical stimuli, and these ratings were replicated after a two-week interval.

\section{Results and Discussion}

To compare the effects of the end stimuli in the various ranges, two measures of discriminability were 
TABLE I

Standard Deviations of Judgments in Each Range Condition for Physical Stimuli

\begin{tabular}{ccccc} 
& \multicolumn{4}{c}{ Range } \\
Stimulus & I & II & III & IV \\
\cline { 2 - 5 } 1 & .00 & .62 & .35 & .00 \\
2 & .26 & .76 & .64 & .26 \\
3 & .26 & .96 & .63 & .46 \\
4 & .52 & .66 & .63 & .63 \\
5 & .59 & .77 & .84 & .51 \\
6 & .80 & .62 & .92 & .52 \\
7 & .46 & .46 & .64 & .52 \\
8 & .82 & .35 & .51 & .00 \\
Mean & .45 & .65 & .64 & .36
\end{tabular}

used. First, we may analyze the variability with which each stimulus in a given range is judged. These data, for physical and social stimuli, are presented in Tables 1 and 2, respectively. We may note that for Ranges I and II, the stimuli in that range with the most salient end anchor are judged less variably than the stimuli in the range with the less salient end stimulus. Thus, Range I of the physical stimuli (Table 1) is judged less variably than Range II, while Range II of the social stimuli (Table 2) is judged less variably than Range I. Further, it can be noted that the average variability of judgements for that range with the least salient end stimulus is about the same as that for the mid-range stimuli of Range III in each stimulus domain. As expected, stimuli in Range IV are judged with the least variability, although it should be noted that in this range condition the intervals between stimuli were necessarily greater than in the other ranges, a factor which undoubtedly contributed to lower variability in judgements. From Tables 1 and 2 it can be observed that for both social and physical stimuli, the end stimulus in that range with the most salient end stimulus (Range I for physical stimuli and Range II for social stimuli) is judged with less variability than any of the other stimuli in Ranges I, II, or III.

While the above analyses are based on the initial, nonreplicated judgements, a second analysis using information measures considers the reliability with which a judge discriminates the stimuli. Tx;y scores (Attneave, 1959) were calculated for each judge. Such a statistic

TABLE 3

Discriminability in Bits $\left(\mathbf{T} \mathbf{x}_{\ominus}^{+} \mathbf{y}\right)$ of Stimuli in Each

Range Condition

Physical Stimuli

\begin{tabular}{cccc}
\multicolumn{4}{c}{ Range } \\
I & II & III & IV \\
\hline 2.58 & 2.48 & 2.31 & 2.64 \\
1.95 & 1.95 & 1.72 & 2.06
\end{tabular}

TABLE 2

Standard Deviations of Judgments in Each Range Condition for Social Stimuli

Stimulus
1
2
3
4
5
6
7
8
Mean

\begin{tabular}{|c|c|c|c|}
\hline \multicolumn{4}{|c|}{ Range } \\
\hline I & II & III & IV \\
\hline 1.28 & 1.62 & 1.64 & 0.59 \\
\hline 1.41 & 1.42 & 1.46 & 0.80 \\
\hline 2.21 & 1.56 & 1.59 & 1.17 \\
\hline 1.53 & 1.51 & 1.85 & 1.32 \\
\hline 1.51 & 1.49 & 2.05 & 1.31 \\
\hline 1.81 & 1.47 & 1.16 & 1.14 \\
\hline 1.81 & 1.37 & 1.71 & 1.32 \\
\hline 1.57 & 1.02 & 1.64 & 0.64 \\
\hline 1.64 & 1.43 & 1.64 & 1.04 \\
\hline
\end{tabular}

indicates the number of reliable discriminations in judging the stimuli in each range. Table 3 presents the mean Tx;y scores for each range of physical and social stimuli. Here we note that the discrimination of Range III, which has no salient end stimuli, is lowest for both social and physical continua. This informational analysis indicates that even though the variability of judgement of stimuli in the mid-range conditions (III) was no less on average than that for the less salient end stimulus conditions (I and II, Tables 1 and 2), mid-range stimuli are judged less reliably in part at least as a result of having no salient end stimuli.

Finally, we may note a major difference between the discriminability of physical and social stimuli from Table 3. It is observed that under the most optimal condtions for discrimination, Range IV, in which two salient end stimuli are present and the inter-stimulus interval is greatest, a mean Tx;y of 2.06, or about four reliable discriminations, is obtained for social stimuli. For physical stimuli, on the other hand, the mean Tx;y of 2.64 corresponds to about six reliable discriminations.

\section{References}

Attneave, F. Applications of information theory to psychology. New York: Holt, 1959.

Bieri, J., Orcutt, B. A., \& Leaman, R. Anchoring effects in sequential clinical judgments. J. abnorm. soc. Psychol., 1963, 67, 616-623.

Hunt, W. A., Schwartz, M. L., \& Walker, R. E. Reliability of clinical judgments as a function of range of pathology. $J$. abnorm. Psychol., 1965, 70, 32-33.

Johnson, D. M., \& King, C. R. Systematic study of end anchoring and central tendency of judgment. J. exp. Psychol., 1964, 67, 501-506.

Miller, H., \& Bieri, J. An informational analysis of clinical judgment.J. abnorm. soc. Psychol., 1963, 67, 317-325.

\section{Note}

1. This research was supported by grant $\mathrm{MH}-08334$ from the National Institute of Mental Health, U. S. Public Health Service, and by grant No. 362, Institute of Social Science, University of California, Berkeley. 